\title{
Contrôle des défauts d'emboutissage par optimisation des efforts serre-flan
}

\section{Application à un panneau de porte}

\author{
Lanouar Ben Ayed* — Arnaud Delamézière* \\ Jean-Louis Batoz* — Catherine Knopf-Lenoir** \\ * Institut Supérieur d'Ingénierie de la Conception \\ Equipe de Recherche en Mécanique et Plasturgie \\ 27 rue d'Hellieule, F-88100 Saint-Dié-des-Vosges \\ \{lanouar.ben-ayed; arnaud.delameziere ; jean-louis.batoz\}@insic.fr \\ ** Université de Technologie de Compiègne, Laboratoire Roberval \\ FRE 2833 UTC-CNRS, BP 20529 \\ F-60205 Compiègne cedex \\ catherine.vayssade@utc.fr
}

\begin{abstract}
RÉSUMÉ. Ce travail concerne l'optimisation des efforts serre-flan en emboutissage, le serreflan étant constitué de zones indépendantes. Les forces optimales appliquées sur ces zones sont calculées pour éviter les défauts d'emboutissage (striction et plissement de la tôle). Dans le cadre de ces travaux, un critère de striction a été formulé. La procédure d'optimisation utilise une surface de réponse basée sur une approximation par moindres carrés pondérés. La méthode est appliquée à un panneau de porte, cas-test de la conférence Numisheet'99.

ABSTRACT. That paper deals with the optimization of the blankholder forces in deep drawing, the blankholder is made of several zones. The optimal forces applied on those zones are computed to avoid defects during the process (necking and wrinkling of the blank). A necking criterion has been formulated. The optimization procedure uses a response surface computed by a moving lest square technique. The methodology is applied to a front door panel, benchmark at the Numisheet'99 conference.

MOTS-CLÉS : emboutissage, striction, plissement, optimisation, force de serrage.

KEYWORDS: deep drawing, necking, wrinkling, optimization, blankholder force.
\end{abstract}

DOI:10.3166/REMN.17.373-395 @ 2008 Lavoisier, Paris 


\section{Introduction}

La simulation numérique du procédé d'emboutissage est suffisamment maîtrisée pour envisager de l'utiliser dans une procédure d'optimisation. L'objectif est de régler de la manière la plus automatique possible les paramètres du procédé pour obtenir une pièce répondant au cahier des charges. Pour cela il faut contrôler les défauts qui peuvent apparaitre lors du procédé comme la striction, qui mène à la rupture, et les défauts géométriques (formation de plis, retour élastique gênant à l'assemblage des pièces embouties).

Régler les paramètres du procédé d'emboutissage consiste à contrôler le mouvement de la tôle pour obtenir la forme souhaitée. Felder (1994) a classé les moyens d'actions, du plus simple au plus difficile à mettre en œuvre :

- la géométrie et la position initiale du flan,

- la force de serrage exercée par le serre-flan, la position et la géométrie des joncs,

- la géométrie de l'outil (pour influer sur le retour élastique),

- la nature et la quantité du lubrifiant,

- le matériau de la tôle,

- la nature de l'outil...

La majorité de ces moyens consiste à gérer le déplacement de la partie de la tôle située sous le serre-flan en ajustant les dimensions du flan, les efforts, la géométrie des joncs et les conditions de lubrification.

Dans notre étude, les variables de conception de la procédure d'optimisation sont les efforts appliqués sur la tôle en différentes zones indépendantes du serre-flan. Suivant la complexité du procédé et le besoin, les valeurs de ces efforts évoluent durant le déplacement du poinçon.

Le but de l'optimisation est de régler les variables de conception pour réaliser des pièces sans défaut. Une part importante de ce travail a été consacrée à la détection numérique des défauts.

Cet article commence par une courte étude bibliographique de l'optimisation en emboutissage. Nous présentons ensuite les critères mis en place pour la détection des défauts. Une procédure d'optimisation utilisant une surface de réponse basée sur les moindres carrés pondérés a été adoptée et utilisée sur une application, l'emboutissage d'un panneau de porte. 


\section{Etude bibliographique de l'optimisation en emboutissage}

Le tableau 1 présente les résultats de l'étude bibliographique de l'optimisation en emboutissage. Il recense, sans prétendre à l'exhaustivité, un certain nombre de travaux, menés essentiellement à partir de simulations numériques.

\begin{tabular}{|c|c|c|c|}
\hline & Variables d'optimisation & Auteurs des travaux de recherche & $\begin{array}{c}\text { Méthode } \\
\text { d'optimisation }\end{array}$ \\
\hline \multirow{17}{*}{ 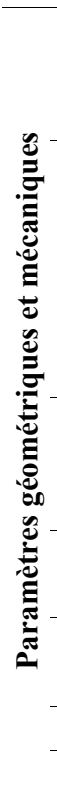 } & \multirow{3}{*}{ épaisseur initiale du flan } & Kleinermann et al., 2003 & $\mathrm{LM}+\mathrm{GC}$ \\
\hline & & Ponthot et Kleinermann, 2006 & $\mathrm{LM}+\mathrm{GC}$ \\
\hline & & Gantar et al., 2005 & $\mathrm{MC}+\mathrm{SR}$ \\
\hline & \multirow{5}{*}{ contour du flan } & Labergère, 2003 & $\mathrm{SR}+\mathrm{AG}$ \\
\hline & & Naceur et al., 2003 & SR \\
\hline & & Barlet, 1998, Naceur, 1998 & $\mathrm{SQP}+\mathrm{AS}$ \\
\hline & & $\begin{array}{l}\text { Ghouati et al., 2000, Gantar et al., } \\
2002\end{array}$ & - \\
\hline & & Keltz, 2004 & $\mathrm{SR}+\mathrm{AS}$ \\
\hline & \multirow{3}{*}{ propriétés du matériau } & Delamézière, 2002 & $\mathrm{SQP}+\mathrm{SR}$ \\
\hline & & Naceur et al., 2004 & $\mathrm{SQP}+\mathrm{AS}$ \\
\hline & & Gantar et al., 2005 & $\mathrm{MC}+\mathrm{SR}$ \\
\hline & géométrie des ioncs & Naceur et al., 2001 & \multirow{2}{*}{$\mathrm{SQP}+\mathrm{AS}$} \\
\hline & geometrie des joncs & Naceur et al., 2004 & \\
\hline & géométrie de la matrice & Keltz, 2004 & $\mathrm{SR}+\mathrm{AS}$ \\
\hline & (rayon...) & Xia et al., 2004, Katayama et al., 2004 & Simplex \\
\hline & géométrie du poinçon & Ohata et al., 1996, Ohata et al., 1998 & Simplex \\
\hline & géométrie du serre-flan & Siegert, 2000, Häussermann, 2000 & - \\
\hline \multirow{11}{*}{ 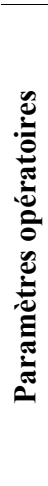 } & \multirow{4}{*}{$\begin{array}{l}\text { force }(\mathrm{s}) \text { de serrage } \\
\text { (pression de serrage) }\end{array}$} & Keltz, 2004 & $\mathrm{SR}+\mathrm{AS}$ \\
\hline & & Hsu et al., 2002, Gantar et al., 2002 & - \\
\hline & & Jansson et al., 2005 & SR \\
\hline & & Gantar et al., 2005 & $\mathrm{MC}+\mathrm{SR}$ \\
\hline & \multirow{2}{*}{ force de retenue (jonc) } & Keltz, 2004 & $\mathrm{SR}+\mathrm{AS}$ \\
\hline & & Naceur et al., 2001 & $\mathrm{SQP}+\mathrm{AS}$ \\
\hline & vitesse d'emboutissage & Ohata et al., 2003 & Simplex + SR \\
\hline & force d'emboutissage & Hsu et al., 2002 & SR \\
\hline & \multirow{2}{*}{$\begin{array}{l}\text { frottement (nature et type } \\
\text { du lubrifiant) }\end{array}$} & Gantar et al., 2002 & - \\
\hline & & Gantar et al., 2005 & $\mathrm{MC}+\mathrm{SR}$ \\
\hline & Température & Ohata et al., 2003 & Simplex + SR \\
\hline
\end{tabular}

LM : Levenberg-Marquadt, GC : gradient conjugué, SR : surface de réponse, AG : algorithme génétique, MC: Monte Carlo et AS: analyse de sensibilité. SQP : programmation quadratique séquentielle

Tableau 1. Etude bibliographique de l'optimisation en emboutissage 
Le classement est effectué par type de paramètres : d'abord ceux de la géométrie et du flan, puis les paramètres opératoires. Les méthodes ou les algorithmes d'optimisation sont également identifiés.

Les travaux d'optimisation en emboutissage ont commencé au milieu des années 1990 (tableau 1) et depuis cette période on note une accélération des publications, qui est due principalement à une augmentation du nombre d'équipes de recherche travaillant sur l'amélioration de la qualité des pièces embouties, à des avancées technologiques des moyens de calcul, des méthodes d'optimisation et des moyens de contrôle et d'ajustement du procédé d'emboutissage etc.

D'après le tableau 1 , on constate que la méthode de surface de réponse est fréquemment utilisée et elle est généralement couplée avec un algorithme d'optimisation de type SQP, GC, AG ou MC. Dans la section 4 une procédure d'optimisation automatique, basée sur la méthode SR et un algorithme d'optimisation itérative de type SQP, est proposée.

\section{Détection des défauts en emboutissage}

D'après (Chu et al., 2001), quatre-vingt pour cent des rebuts d'emboutissage de pièces destinées au secteur de l'automobile sont dus aux plis, les vingt pour cent restants sont dus aux déchirures. Différents critères sont utilisés pour prédire numériquement l'apparition de ces défauts.

\subsection{Détection du risque de striction}

La striction est la localisation de la déformation dans une petite zone de la tôle, qui est alors fragilisée : une rupture risque donc de se produire.

Plusieurs méthodes ont été développées pour prédire numériquement ou expérimentalement le risque de striction. Expérimentalement (Col, 2002) Keeler et Goodwin ont proposé au début des années 1960, l'utilisation des courbes limites de formage (CLF). Boudeau et Gélin (2000) ont mené une recherche bibliographique sur la prédiction de la CLF. Les différentes approches sont séparées en deux catégories. Une considère la matière comme un milieu continu homogène et l'autre comme un milieu continu hétérogène. Pour un milieu continu homogène, la striction est décrite comme un phénomène d'instabilité plastique. La détection se fait en utilisant par exemple la technique de perturbation de l'état d'équilibre (Dudzinski et Molinari, 1990 ; Boudeau et Gélin, 2000). L'état de déformation est souvent considéré plan au moment de la striction (Hora et al., 1996 ; Brunet et al., 1998). Si nous considérons la matière comme un milieu continu hétérogène, la striction est considérée comme un problème de bifurcation ou comme provenant d'un défaut géométrique où toute la déformation va se produire (Marciniak et Kuczynski, 1967 ; Graf et Hosford, 1990). 
Trois méthodes considèrent un milieu homogène. La méthode ALS (analyse linéaire de stabilité) proposée initialement par (Molinari, 1985) a été utilisée et améliorée suite à de nombreux travaux: (Boudeau, 2003 ; Lejeune, 2002 ; Keltz, 2004). Le concept de base de cette approche est fondé sur l'observation de la réponse d'un système stable suite à une petite perturbation locale. Le phénomène de la striction localisée est traité comme un problème d'instabilité de l'équilibre mécanique local.

La seconde méthode, développée par (Ladevèze, 1981; Cordebois, 1983 ; Mouric, 2002), traite le phénomène de striction comme un problème d'instabilité plastique.

Lorsqu'un solide est soumis à un chargement externe croissant, un champ de vitesses se développe dans ce dernier. Une fois que le chargement s'arrête (la charge externe étant maintenue constante), deux cas peuvent se manifester :

- en tout point du solide le champ de vitesses est nul. Son état est donc stable et il n'y a pas de risque de striction,

- un ou plusieurs points du solide ont une vitesse non nulle, l'état en ces points est instable et il y a un risque de rupture.

Le phénomène de striction se produit au moment où la solution d'un problème d'équilibre décrit sous forme variationnelle, devient instable. C'est pourquoi il est considéré comme un problème d'instabilité plastique.

La troisième méthode choisie dans ce travail est le critère modifié de force maximale (CMFM). Suite aux travaux de Considère (1885) et Swift (1952), Hora et al., (1996) calculent la contrainte principale maximale $\sigma_{1}$ dépendant de la déformation principale maximale $\varepsilon_{1}$ et du taux de déformation $\beta$, dans le cas d'un état de contraintes de traction biaxiale :

$$
\beta=\frac{\Delta \varepsilon_{2}}{\Delta \varepsilon_{1}}
$$

où $\Delta \varepsilon_{2}$ est l'accroissement de la déformation principale minimale.

Le critère d'instabilité proposé par Hora est :

$$
\sigma_{1}^{\mathrm{cr}}=\frac{\partial \sigma_{1}}{\partial \varepsilon_{1}}+\frac{\partial \sigma_{1}}{\partial \beta} \frac{\mathrm{d} \beta}{\mathrm{d} \varepsilon_{1}} \leq \sigma_{1}
$$

Dans ce travail le CMFM a été implémenté dans le code de calcul Abaqus pour calculer la contrainte critique $\sigma_{1}^{\text {cr }}$ à chaque pas de temps et à chaque point d'intégration en tenant compte de l'historique de l'état de déformation. Un indicateur 
de risque de striction $\mathrm{R}^{\mathrm{el}}$ est défini comme le rapport de la contrainte actuelle et de la contrainte critique.

$$
\mathrm{R}^{\mathrm{el}}=\sigma_{1} / \sigma_{1}^{\mathrm{cr}}
$$

Il y a risque de striction si $\mathrm{R}^{\mathrm{el}} \geq 1$. Cet indicateur a été validé sur des pièces tests (godet cylindrique et test de Nakazima) en comparaison avec des expériences (Ben Ayed et al., 2006).

Dans le contexte de l'optimisation une fonction limitation est définie pour restreindre cet indicateur à une valeur maximale notée $\mathrm{R}_{\mathrm{r}}^{\text {limite }}$

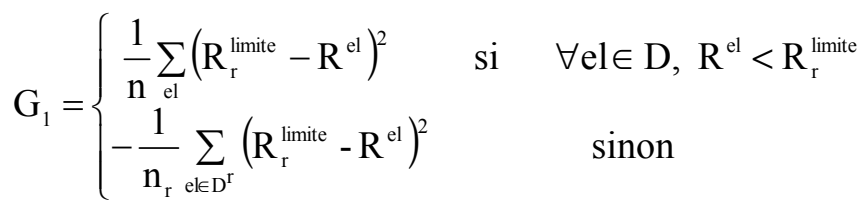

avec D l'ensemble de tous les (n) éléments de la tôle et $D^{r}$ l'ensemble des $\left(n_{r}\right)$ éléments qui ont un indicateur de striction, $\mathrm{R}^{\mathrm{el}}$, supérieur à $\mathrm{R}_{\mathrm{r}}^{\text {limite }}$.

\subsection{Détection du risque de plissement}

\subsubsection{Plissement dans la partie utile}

Le phénomène de plissement a fait l'objet de nombreux travaux. Hutchinson et $a l$. , (1985) ont développé un critère basé sur la méthode d'analyse locale de bifurcation plastique de Hill, afin d'évaluer le risque de plissement. Ensuite, Neale et al., (1990) et Amézianne-Hassani et al., (1991) ont montré que l'état critique de ce phénomène dépend de la courbure locale de la tôle. La méthode d'analyse locale proposée par Hutchinson et Neale a été reprise, simplifiée et appliquée sur des pièces industrielles par (Brunet et al., 1997). Ces critères ne peuvent être appliqués que sur des surfaces libres avec courbure, car les interactions entre les outils et la tôle ont été négligées.

Pour estimer le risque de plissement, Hutchinson, Neale et Tugcu ont proposé les hypothèses suivantes :

- la configuration déformée est une coque à courbure constante et d'épaisseur constante,

- l'état de contraintes est plan, uniforme et de membrane (décrit par deux contraintes principales),

- le phénomène de flambage plastique est décrit par le critère de bifurcation plastique de Hill, 
- la théorie de la déformation plastique totale est appliquée au flambement,

- l'interaction outils-pièce est négligée,

- le plissement est localisé (l'influence des dimensions de la coque et des conditions aux limites est omise),

- les plis sont de faible longueur d'onde: la théorie des coques minces surbaissées de Donnell-Mushtari-Vlasov est retenue.

Brunet et al., (1997) ont ajouté l'hypothèse supplémentaire :

- le rapport des contraintes principales reste constant entre l'état de préflambage et l'étape de bifurcation plastique.

Le problème à résoudre reste cependant fortement non linéaire, et nécessite une résolution itérative coûteuse en temps sans garantie de convergence.

Pour une analyse qualitative une hypothèse supplémentaire est utilisée :

- les axes principaux des courbures et ceux des contraintes principales sont confondus.

Le calcul analytique d'une contrainte critique $\sigma_{1}^{c r}$ est alors effectué. Pour un état de contrainte défini par le rapport :

$$
\alpha=\frac{\sigma_{2}}{\sigma_{1}}
$$

et connaissant la géométrie par l'épaisseur de la tôle $\mathrm{h}$ et les rayons de courbure $\mathrm{R}_{1}$ et $\mathrm{R}_{2}$, nous calculons alors, pour une contrainte $\sigma_{2}$ connue, la contrainte critique $\sigma_{1}^{c r}$ par : (Brunet et al., 1997)

$$
\sigma_{1}^{\mathrm{cr}}=\mathrm{K}\left[\frac{1+\mathrm{r}}{\sqrt{3(1+2 \mathrm{r})}}\left(\frac{\mathrm{h}}{\mathrm{R}_{2}}\right) \sqrt{\mathrm{n}_{\mathrm{c}}}\left(\alpha^{\prime}\right)^{1-1 / \mathrm{n}}\right]^{\mathrm{n}} \text { avec } \alpha^{\prime}=\sqrt{1-\frac{2 \mathrm{r} \alpha}{1+\mathrm{r}}+\alpha^{2}}
$$

où $\mathrm{K}$ est le seuil de résistance plastique, $\mathrm{n}_{\mathrm{c}}$ le coefficient d'écrouissage dans la loi d'Hollomon et $\mathrm{r}$ le coefficient moyen d'anisotropie.

Pour un élément fini le risque de plissement est défini par :

$$
\mathrm{R}_{\mathrm{p}}^{\mathrm{el}}=\frac{\sigma_{1}}{\sigma_{1}^{\mathrm{cr}}}
$$

où $\sigma_{1}$ est la contrainte principale de compression au point considéré. 
Un risque de plissement existe quand $\mathrm{R}_{\mathrm{p}}^{\mathrm{el}}$ est supérieur à 1. Les hypothèses de ce critère imposent qu'il ne peut pas être utilisé pour la partie de la tôle sous serre-flan.

Une fonction limitation est définie :

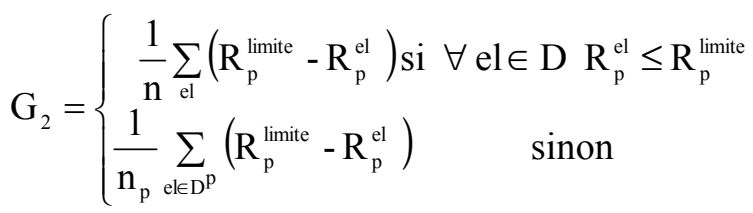

avec $D^{p}$ l'ensemble des $n_{p}$ éléments, qui ont un indicateur de plissement, $R_{p}^{e l}$, supérieur à $\mathrm{R}_{\mathrm{p}}^{\text {limite }}$.

\subsubsection{Plissement sous serre-flan}

Le critère précédent néglige toute interaction entre les outils et la tôle et suppose que la tôle présente une courbure. Il n'est donc pas utilisable sous le serre-flan. Labergère (2003) a formulé un autre critère basé sur des considérations purement géométriques. Il écrit une fonction limitation dépendant de l'angle d'inclinaison entre deux éléments connexes du maillage dans une zone serre-flan. Keltz (2004) utilise une fonction basée sur la distance entre le flan et la matrice ou encore une fonction prenant en compte l'évolution des courbures d'éléments proches.

Dans ce travail, le plissement sous serre-flan est détecté en analysant l'inclinaison de chaque élément fini par rapport à la surface serre-flan. Une fonction limitation a été formulée afin d'éviter la formation de fortes ondulations sous le serre-flan qui sont la cause d'une usure rapide des outils. Elle est calculée en fonction de l'angle d'inclinaison, $\theta$, des éléments sous serre-flan par rapport à la surface horizontale de la matrice (figure 1):

$$
\mathrm{G}_{3}=\left\{\begin{array}{cl}
\frac{1}{\mathrm{n}_{\mathrm{s}}} \sum_{\mathrm{el}}\left(\sin \left(\theta_{\max }\right)-\sin \left(\theta_{\mathrm{el}}\right)\right) & \text { si } \forall \text { el } \in \mathrm{D}^{\mathrm{SF}} \quad \theta_{\mathrm{el}}<\theta_{\text {max }} \\
\sum_{\mathrm{el} \in \mathrm{D}^{\mathrm{pl}}}\left(\sin \left(\theta_{\text {max }}\right)-\sin \left(\theta_{\mathrm{el}}\right)\right) & \text { sinon }
\end{array}\right.
$$

avec $\mathrm{D}^{\mathrm{SF}}$ l'ensemble des $\mathrm{n}_{\mathrm{s}}$ éléments sous serre-flan à la fin de l'opération d'emboutissage et $\mathrm{D}^{\mathrm{pl}}$ l'ensemble des éléments sous serre-flan qui ont un angle d'inclinaison supérieur à $\theta_{\max }$. 


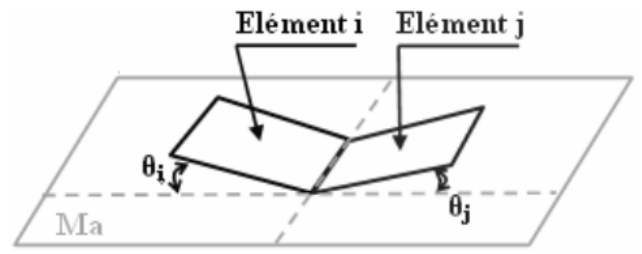

Figure 1. Détection des plis sous serre-flan

\section{Procédure d'optimisation}

Le temps de calcul d'une simulation du procédé d'emboutissage en dynamique explicite est de l'ordre de grandeur d'une heure pour une pièce simple et de plusieurs heures pour une pièce industrielle. La procédure d'optimisation doit donc minimiser le nombre d'appels à la simulation numérique et privilégier les possibilités de faire des calculs en parallèle.

Ce problème s'est posé pour l'ensemble des équipes de recherche participant au projet RNTL OPTIMAT «Optimisation des Procédés de TransformatIon des MATériaux ». Un consensus s'est dégagé autour de l'utilisation de métamodèles, de surfaces de réponse ou de réseaux de neurones. Ces méthodes visent à construire une approximation des fonctions objectives et limitations avant de résoudre le problème d'optimisation ainsi « approché ».

La procédure d'optimisation (figures 3 et 4 ) choisie ici est basée sur l'utilisation d'un espace de recherche adaptatif, de la méthode de surface de réponse et d'un algorithme d'optimisation de type SQP (sequential quadratic programming).

Un premier espace de recherche, définissant le domaine d'étude dans lequel varient les variables de conception, est établi après une phase d'exploration. Les fonctions objectif et limitations sont ensuite évaluées dans un nombre limité de points définis en utilisant un plan d'expérience composite à face centrée (Goupy, 1999). L'optimum est localisé en utilisant un processus d'optimisation basé sur une série d'actualisations du problème d'optimisation approché (globale/locale) au voisinage des optima obtenus. Une fois que l'optimum est déterminé un nouvel espace de recherche est alors défini dans son voisinage et la procédure d'optimisation est répétée jusqu'à ce que les deux derniers optima $x_{\mathrm{opt}}^{\mathrm{D}_{\mathrm{J}}}$ et $x_{\mathrm{opt}}^{\mathrm{D}_{\mathrm{J}}+1}$ soient très proches [10] ou bien jusqu'à ce que le domaine de recherche devienne très petit [11] (Condition $n^{\circ} 2$, figures 2 et 3$)$ :

$$
\begin{aligned}
& \left\|x_{\mathrm{opt}}^{\mathrm{D}_{\mathrm{J}+1}}-x_{\mathrm{opt}}^{\mathrm{D}_{\mathrm{J}}}\right\| \leq \frac{\Delta x^{\min }}{l} \\
& \left|x_{i}^{\max }-x_{i}^{\min }\right| \leq \Delta x^{\min } \quad \forall i=1, \ldots, n_{v}
\end{aligned}
$$


où $n_{v}$ est le nombre de variable, $\Delta x^{\min }$ est le pas minimal de l'espace de recherche entre les bornes inférieure $x_{i}^{\min }$ et supérieure $x_{i}^{\max }$ de la $\mathrm{i}^{\mathrm{e}}$ variable, et $l$ le nombre minimal de niveaux dans le plan d'expérience.

Il est à noter que le plan composite à face centrée utilise trois niveaux pour chaque variable.

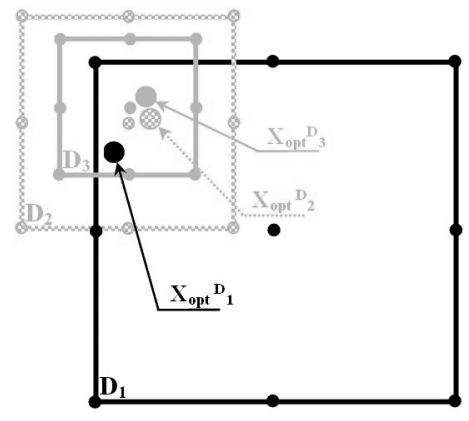

Figure 2. Actualisation de l'espace de recherche (2D)

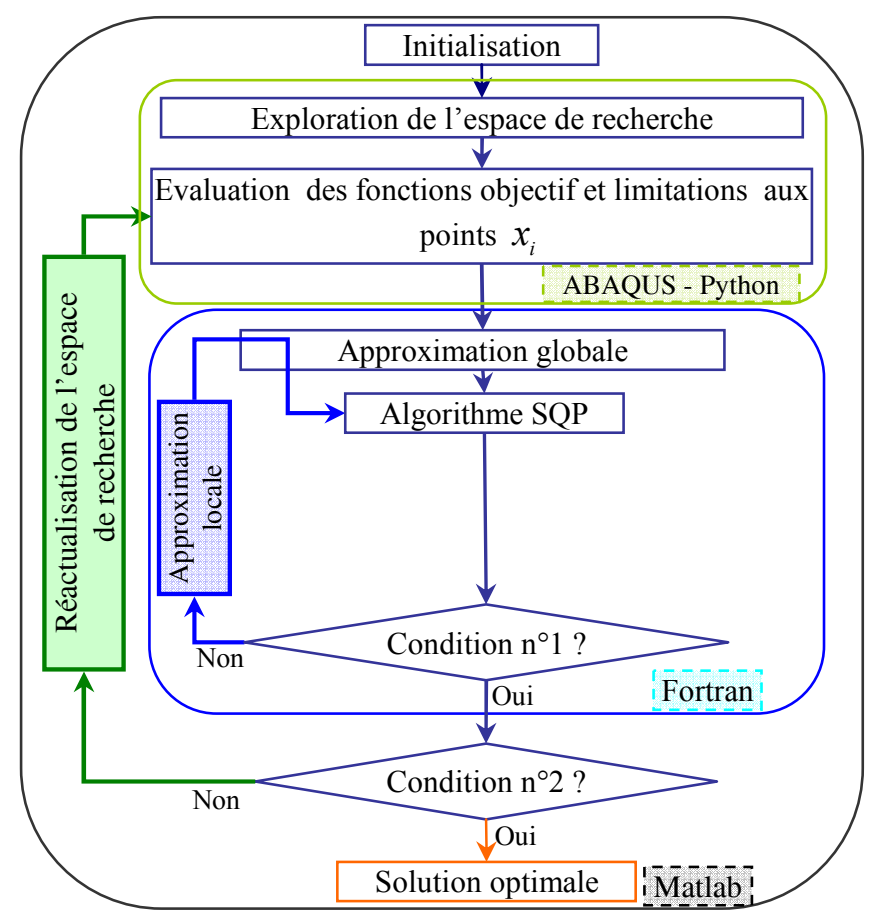

Figure 3. Procédure d'optimisation 
Pour faciliter la convergence de l'algorithme d'optimisation, la fonction objectif a été normalisée sur l'intervalle $[0,1]$ et les fonctions limitations sont normalisées sur l'intervalle $[-1,1]$. Ces fonctions ont été écrites explicitement en utilisant la méthode de moindres carrés pondérés également appelée approximation diffuse (Villon, 1991) :

$$
\widetilde{F}(x)=p^{T}(x) \cdot a(x)
$$

où $p^{T}(x)$ est une base polynomiale d'ordre 2 et $a(x)$ le vecteur des coefficients, qui sont déterminés en minimisant le résidu :

$$
R=\sum_{i=1}^{n_{e}} w\left(x-x_{i}\right)\left(\widetilde{F}\left(x_{i}\right)-F\left(x_{i}\right)\right)^{2}
$$

où $n_{e}$ est le nombre de points d'évaluations $x_{i}, F\left(x_{i}\right)$ la valeur de la fonction en ces points et $w\left(x-x_{i}\right)$ la fonction d'influence (ou poids) définie comme suit :

$$
w\left(x-x_{i}\right)= \begin{cases}\left(e^{-\left(\frac{\left\|x-x_{i}\right\|}{\alpha \cdot d}\right)^{2}}-e^{-\frac{1}{\alpha^{2}}}\right) /\left(1-e^{-\frac{1}{\alpha^{2}}}\right) & \text { si }\left\|x-x_{i}\right\| \leq d \\ 0 & \text { sinon }\end{cases}
$$

où $d$ est la distance limitant le domaine de validité de l'approximation (ou le domaine d'influence de la fonction poids) et le paramètre $\alpha$ contrôle la courbure de la fonction poids. $\alpha$ est fixé à $1 / 3$.

La fonction poids est toujours positive. Sa valeur décroît avec la distance entre le point d'évaluation $x_{i}$ auquel elle est associée et le point $x$, où la fonction est approchée. Elle est égale à l'unité quand $x=x_{i}$ et à zéro en dehors du domaine de validité qui est défini par $d$.

Afin que le processus d'optimisation localise la zone où se trouve l'optimum global et évite les optima locaux du problème d'optimisation, une approximation globale (figures 3 et $4 \mathrm{a}$ ) est construite dans un premier temps en utilisant la méthode de moindres carrés $\left(w\left(x-x_{i}\right)=1\right)$. Une série d'approximations locales sont ensuite construites au voisinage des optima en utilisant la méthode diffuse afin d'améliorer la précision. Durant la progression du processus d'optimisation (Figure 4b) le domaine de validité est ajusté, centré et rétréci de plus en plus autour de l'optimum en réduisant la distance $d$ comme suit : 


$$
d_{k+1}= \begin{cases}d_{\min } & \text { si } d_{k}\left|\left(\frac{k}{k+1}\right)^{2}-1\right| \leq d_{\text {min }} \\ d_{k}\left|\left(\frac{k}{k+1}\right)^{2}-1\right| & \text { sinon }\end{cases}
$$

où $d_{\min }$ est la distance minimale à respecter. Il est à noter que le nombre de points d'évaluations à l'intérieur du domaine de validité doit être toujours supérieur au nombre des coefficients à déterminer.

L'actualisation des approximations est stoppée quand les deux derniers optima $x_{\mathrm{opt}}^{\mathrm{k}}$ obtenus sont très proches (Condition $\mathrm{n}^{\circ} 1$, figures 3 et $4 \mathrm{~b}$ ) :

$$
\left\|x_{\mathrm{opt}}^{\mathrm{k}+1}-x_{\mathrm{opt}}^{\mathrm{k}}\right\| \leq 10^{-4}
$$

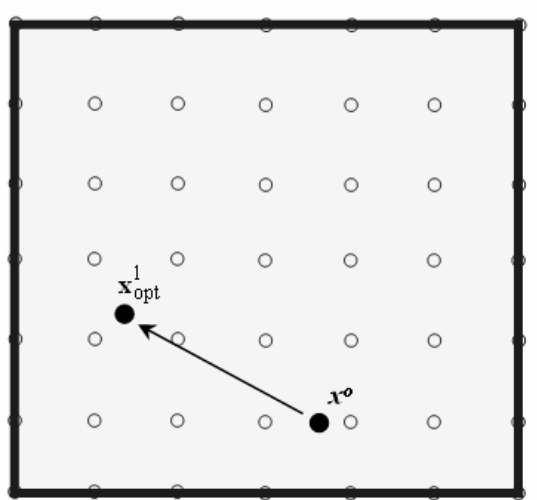

(a)

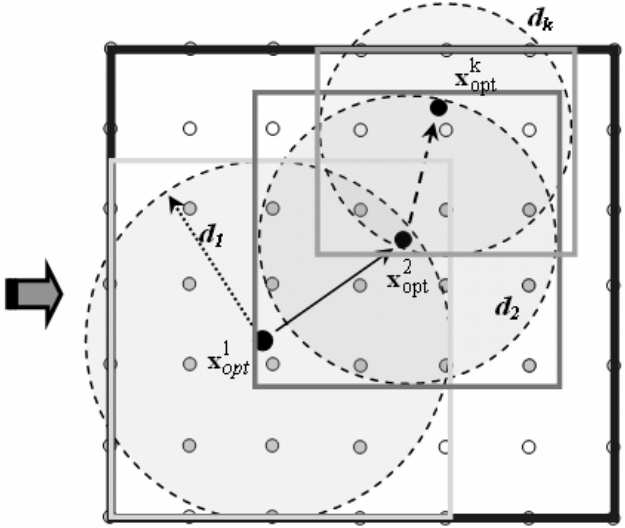

(b)

Domaine de validité de la première approximation globale

_ Domaine de validité de la première approximation locale

- Domaine de validité de la seconde approximation locale Domaine de validité de la $\mathrm{k}^{\text {ème }}$ approximation locale

Figure 4. Processus de résolution du problème d'optimisation approché pour un plan d'expérience donné : approximation globale (a) et approximations locales

\section{Application à un panneau de porte}

\subsection{Données du cas-test (Numisheet'99)}

La pièce étudiée (figure 5), un panneau de porte avant, est réalisée en quatre opérations : emboutissage, découpage et deux opérations de bridage. Quatre types de 
matériaux ont été proposés, trois aciers (DDQ, HLS et BH220) et un aluminium (6016T4). Les paramètres opératoires du procédé et les propriétés mécaniques de l'acier DDQ sont les suivants :

- le coefficient de frottement de Coulomb est de 0,15 ;

- le module d'Young E est égal à $220 \mathrm{GPa}$;

- la masse volumique est égale à $7800 \mathrm{~kg} / \mathrm{m} 3$;

- le coefficient de Poisson vaut 0,3 ;

- les coefficients d'anisotropies sont $r_{0}=1,73, r_{45}=1,23, r_{90}=2,02$;

- la loi d'écrouissage de Swift est exprimée sous la forme suivante: $\sigma=521,16\left(0,00626+\varepsilon_{p}\right)^{0,214} \mathrm{MPa}$

- la force de serrage initiale proposée est égale à $300 \mathrm{kN}$;

- la vitesse de déplacement du poinçon et du serre-flan est égale à $450 \mathrm{~mm} / \mathrm{s}$.

La tôle a les dimensions $750 \times 950 \mathrm{~mm}$ et une épaisseur de $1 \mathrm{~mm}$. Seule l'opération d'emboutissage est considérée. Sur la figure 5 sont présentés les outils (poinçon, matrice et serre-flan), le flan et les plans de coupe sur lesquels des résultats numériques et expérimentaux sont disponibles.
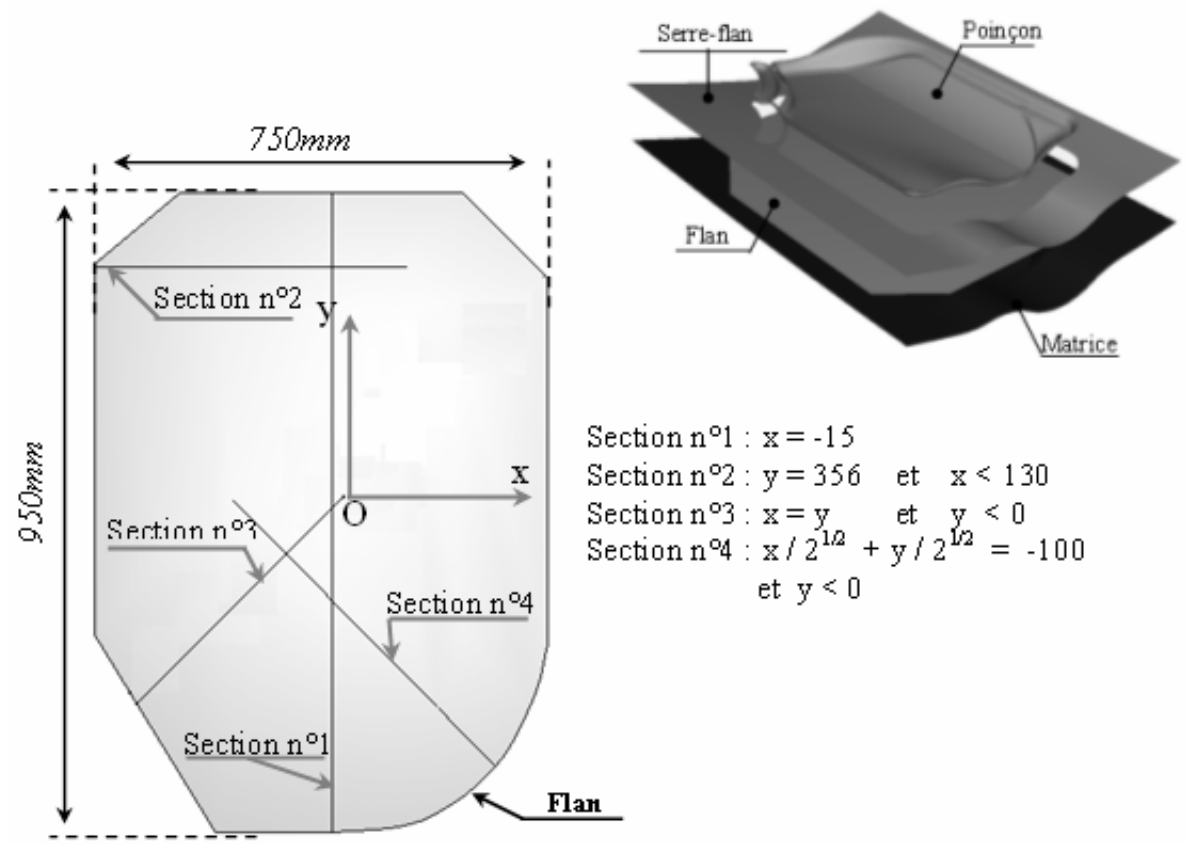

Section $\mathrm{n}^{\circ} 1: \mathrm{x}=-15$

Section $n^{\circ} 2: y=356$ et $x<130$

Section $\mathrm{n}^{\circ} 3: \mathrm{x}=\mathrm{y}$ et $\mathrm{y}<0$

Section $n^{\circ} 4: x / 2^{1 / 2}+y / 2^{1 / 2}=-100$

et $\mathrm{y}<0$

Figure 5. Outils, flan et plans (sections) de coupe 
$\mathrm{Au}$ niveau de la simulation numérique, cette application regroupe quelques difficultés marquantes, à savoir un serre-flan dont la surface n'est pas plane et la présence de joncs. Etant donné que la surface de la matrice n'est pas plane, nous avons utilisé dans un premier temps la gravité pour stabiliser le flan sur la matrice et être plus proche de la réalité. Ensuite, le serrage et l'emboutissage (descente du poinçon) sont simulés dans une seconde étape.

La modélisation de l'opération d'emboutissage a été effectuée en utilisant une formulation dynamique explicite (double précision) et sans tenir compte des joncs. L'objectif de ce travail est d'utiliser un serre-flan à plusieurs zones de réglage à la place des joncs de serrage et de minimiser le travail des efforts extérieurs fournis au système outils-flan.

\subsection{Paramètres numériques du modèle aux éléments finis}

La procédure d'optimisation nécessite de lancer plusieurs simulations pour différents jeux de variables de conception (efforts serre-flan). Pour obtenir les résultats le plus rapidement possible, les paramètres numériques du modèle sont réglés à partir d'essais préliminaires, et le degré de précision de ces résultats est ensuite vérifié.

Les surfaces d'outils sont maillées sur des considérations de bonne représentation géométrique. La tôle est maillée finement dans les zones où elle va fortement se déformer. La vitesse du poinçon est réglée à $1.35 \mathrm{~ms}^{-1}$ pour diminuer le temps de calcul. Le temps CPU total, pour les trois opérations, gravité, serrage et emboutissage est de $18 \mathrm{~h}$. En utilisant l'option RESTART (HKS, 2005) disponible sous Abaqus, afin de répéter uniquement l'étape de serrage et d'emboutissage sans refaire l'étape de gravité, le temps CPU a été limité à $13 \mathrm{~h}$.

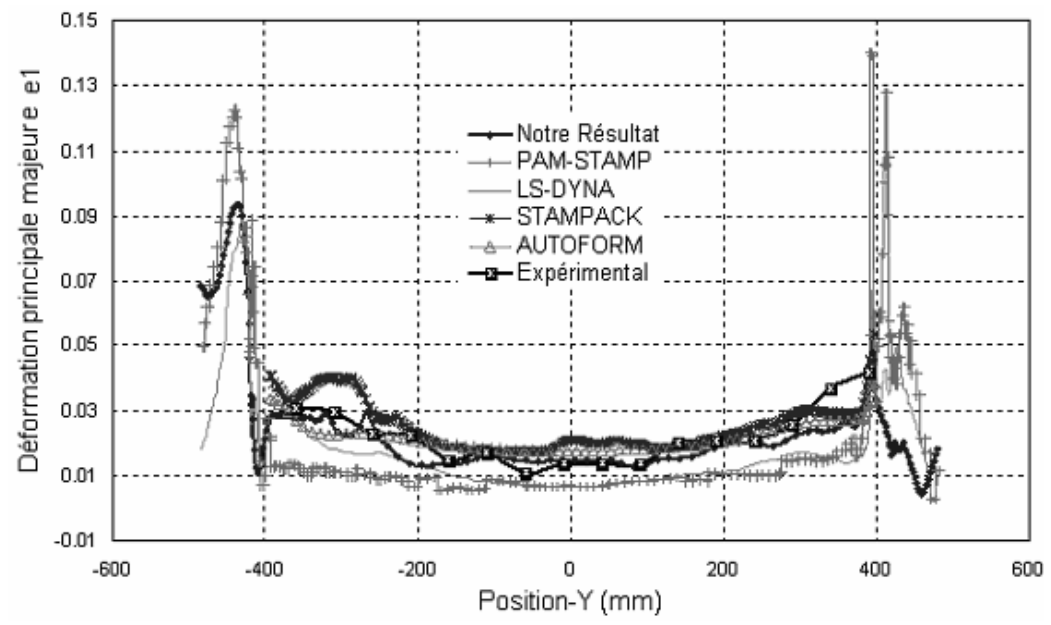

Figure 6. Comparaison et validation du modèle numérique (section $\left.n^{\circ} 1\right)$ 
Les résultats obtenus sont comparés avec ceux publiés lors de la conférence Numisheet'99. Sur la figure 6, nous présentons l'évolution de la déformation principale maximale suivant la section $n^{\circ} 1$. Elle est en bonne concordance avec les résultats expérimentaux et numériques obtenus par différents codes. Les résultats obtenus dans les régions extrêmes ne sont pas corrects car les joncs ne sont pas pris en compte dans notre modélisation.

\subsection{Optimisation des efforts serre-flan}

\subsubsection{Le problème}

Sept surfaces de serre-flan ont été choisies. Le serre-flan a été décomposé en sept zones de serrage (figure 7). Pour chacune d'elles, la force de serrage est supposée constante durant l'opération d'emboutissage. Ces surfaces ont été choisies de manière à améliorer l'écoulement de la matière sous le serre-flan et la qualité de l'embouti, en se basant sur les résultats de calculs obtenus en termes d'épaisseur, d'avalement de la matière et de répartition des plis sous serre-flan :

- pour mieux contrôler l'écoulement de la matière dans les coins d'entrée de la matrice, quatre serre-flans ont été choisis $\left(\mathrm{BH}^{\circ} 1, \mathrm{BH}^{\circ} 3, \mathrm{BH}^{\circ} 5\right.$ et $\left.\mathrm{BH} \mathrm{n}^{\circ} 6\right)$,

- le serre-flan $\left(\mathrm{BH}^{\circ} 2\right)$ a été choisi afin de faciliter l'avalement de la matière dans la zone qui lui correspond et d'éviter ainsi le risque de rupture dans la partie utile de la pièce où nous avons enregistré un amincissement maximal de l'ordre de $20,6 \%$ avec l'effort de serrage initial $(300 \mathrm{kN})$.

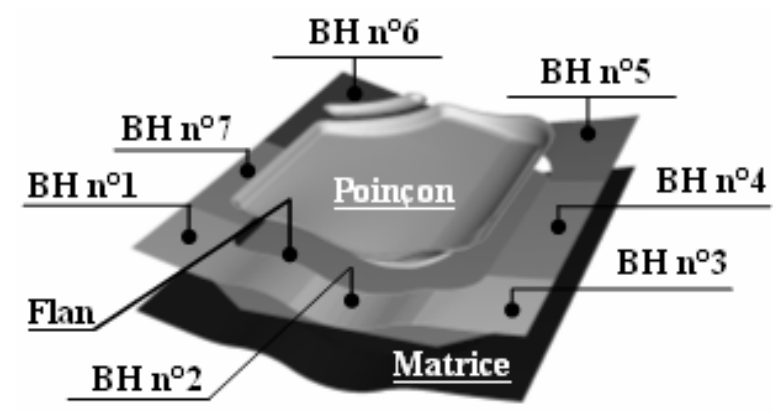

Figure 7. Les sept zones du serre-flan

Comme indiqué précédemment un plan d'expérience de type étoile composite à face centrée a été utilisé (avec 3 niveaux pour chaque variable). Pour $n_{v}$ variables indépendantes, le plan composite nécessite $2^{n_{v}}+2 n_{v}+1$ évaluations des fonctions objectives et limitations, soit 143 simulations pour sept efforts de serrage. 
La réalisation de ce plan d'expérience complet, nécessiterait donc 77 jours et 11 heures de calcul. Dans le cas où on réactualise l'espace de recherche trois fois il nous faudrait 232 jours et 9 heures de calcul avant d'atteindre l'optimum global avec précision. Pour diminuer ce temps, nous avons choisi de décomposer l'optimisation en deux sous-problèmes. Dans le premier, nous optimisons uniquement quatre efforts $\left(\mathrm{BH} \mathrm{n}^{\circ} 1, \mathrm{BH} \mathrm{n}^{\circ} 2, \mathrm{BH} \mathrm{n}^{\circ} 3\right.$ et $\left.\mathrm{BH} \mathrm{n}^{\circ} 4\right)$. Les serre-flans 5 , 6 et 7 sont regroupés en un seul serre-flan, sur lequel nous avons imposé un effort de serrage de $100 \mathrm{kN}$. Une fois que ce sous-problème est résolu nous passons au deuxième problème d'optimisation portant sur les 3 efforts restants et en utilisant les quatre valeurs optimales des quatre efforts optimaux obtenus dans le premier sousproblème. Pour un plan d'expérience de type composite à face centrée et avec 4 variables nous devons effectuer 25 analyses et 15 pour le cas de 3 variables. Cette stratégie nous a permis ainsi de réduire le nombre de calculs nécessaires. En supposant trois réactualisations de l'espace de recherche tant pour le problème d'optimisation initial que pour le problème approché ( 3 fois pour chaque sousproblème) le nombre de calculs nécessaires passe de $429(=3 \mathrm{x} 143)$ à seulement $120(=3 \times(25+15))$.

Sans doute ne trouverons-nous pas exactement les mêmes efforts optimaux en résolvant ces deux problèmes séquentiellement mais ils sont $a$ priori très proches les uns des autres et cela, pour deux raisons :

i. les serre-flans $\left(\mathrm{BH} \mathrm{n}^{\circ} 5, \mathrm{BH} \mathrm{n}^{\circ} 6\right.$ et $\left.\mathrm{BH} \mathrm{n}{ }^{\circ} 7\right)$ ont été regroupés parce qu'ils sont les plus éloignés de la zone où il y a un risque de rupture, et donc ils n'ont pas une influence significative sur l'amincissement maximal ;

ii. la force du serre-flan équivalent $\left(\mathrm{BH}_{-} \mathrm{n}^{\circ} 5 \mathrm{n}^{\circ} 6 \mathrm{n}^{\circ} 7\right)$ a été choisie de façon à ce qu'elle ne contribue pas à la réduction du risque de plissement sous les serreflans adjacents. De très faibles ondulations sont ainsi acceptées sous ce dernier.

\subsubsection{Résultats et discussion}

Nous rappelons que le contrôle de la rupture se fait par l'indicateur $\mathrm{R}^{\mathrm{el}}$ de la striction localisée défini en se basant sur le CMFM [4]. La valeur limite $\mathrm{R}_{\text {limite }}^{\mathrm{el}}$ de cet indicateur a été fixée à 0,975 pour cette application. Le contrôle de plissement sous serre-flan se fait par l'évaluation de l'angle d'inclinaison des éléments sous serreflan par rapport à la surface de la matrice. L'angle d'inclinaison maximum $\theta_{\max }$ a été limité à 1 degré. Concernant le plissement dans la partie utile de la pièce, l'indicateur $\mathrm{R}_{\mathrm{p}}^{\mathrm{el}}$ a été limité à 4,5.

Les valeurs optimales des efforts de serrage obtenues à chaque réactualisation, sont reportées sur le tableau 2 et les efforts sur la figure 8 . 


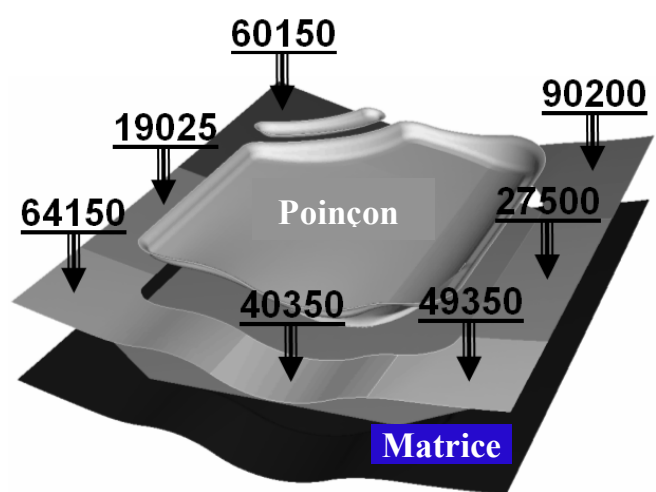

Figure 8. Efforts optimaux pour le panneau de porte

\begin{tabular}{|c|c|c|c|c|c|c|}
\hline $\mathrm{BH}$ & Plan $n^{\circ} 1$ & $\begin{array}{l}\text { Optimum } \\
\text { plan } n^{\circ} 1\end{array}$ & Plan $n^{\circ} 2$ & $\begin{array}{l}\text { Optimum } \\
\text { plan } n^{\circ} 2\end{array}$ & Plan $n^{\circ} 3$ & $\begin{array}{l}\text { Optimum } \\
\text { plan } n^{\circ} 3\end{array}$ \\
\hline \multicolumn{7}{|c|}{ Sous-problème $n^{\circ} 1$} \\
\hline $\mathrm{n}^{\circ} 1$ & $50 \leq \mathrm{F} \leq 80$ & 59,15 & $49,15 \leq \mathrm{F} \leq 69,15$ & 69,15 & $64,15 \leq \mathrm{F} \leq 74,15$ & 64,15 \\
\hline $\mathrm{n}^{\circ} 2$ & $20 \leq \mathrm{F} \leq 50$ & 27,95 & $17,95 \leq \mathrm{F} \leq 37,95$ & 35,35 & $30,35 \leq \mathrm{F} \leq 40,35$ & 40,35 \\
\hline$n^{\circ} 3$ & $40 \leq \mathrm{F} \leq 70$ & 44,35 & $34,35 \leq \mathrm{F} \leq 54,35$ & 54,35 & $49,35 \leq \mathrm{F} \leq 59,35$ & 49,35 \\
\hline$n^{\circ} 4$ & $20 \leq \mathrm{F} \leq 50$ & 30,05 & $20,05 \leq \mathrm{F} \leq 40,05$ & 27,85 & $22,85 \leq \mathrm{F} \leq 32,85$ & 27,50 \\
\hline \multicolumn{7}{|c|}{ Sous-problème $n^{\circ} 2$} \\
\hline $\begin{array}{l}\mathrm{n}^{\circ} 5 \\
(\mathrm{kN})\end{array}$ & $80 \leq \mathrm{F} \leq 110$ & 102,65 & $92,65 \leq \mathrm{F} \leq 112,65$ & 92,65 & $87,65 \leq \mathrm{F} \leq 97,65$ & 90,20 \\
\hline $\mathrm{n}^{\circ} 6$ & $50 \leq \mathrm{F} \leq 80$ & 72,65 & $62,65 \leq \mathrm{F} \leq 82,65$ & 62,65 & $57,65 \leq \mathrm{F} \leq 67,65$ & 60,15 \\
\hline $\mathrm{n}^{\circ} 7$ & $15 \leq \mathrm{F} \leq 45$ & 29,025 & $19,02 \leq \mathrm{F} \leq 39,02$ & 19,10 & $14,00 \leq \mathrm{F} \leq 24,00$ & 19,02 \\
\hline
\end{tabular}

Tableau 2. Résultats d'optimisation pour chaque sous-problème et chaque plan d'expérience $(k N)$ 
La distribution de l'épaisseur est représentée sur la figure 9 avant optimisation et sur la figure 10 après optimisation. Nous remarquons que les ondulations sous serreflan ont disparu après optimisation et que l'épaississement a été limité à $1,1 \mathrm{~mm}$ $(+10 \%)$ au lieu de $1,149 \mathrm{~mm} \quad(+15 \%)$ avant l'optimisation. Cependant, l'amincissement a augmenté légèrement, il est passé de $20,6 \%(0,794 \mathrm{~mm})$ à $21,2 \%$ $(0,788 \mathrm{~mm})$ et le critère de striction reste bien inférieur à 1 . Du côté énergétique, on a réussi à réduire l'énergie externe de $1,6 \%(-252 \mathrm{~kJ})$, bien que la somme des efforts sur les serre-flans $(351 \mathrm{kN})$ soit supérieure à l'effort de serrage initial $(300 \mathrm{kN})$. Ce fait montre qu'une répartition optimale des efforts de serrage permet de contrôler, d'améliorer et de faciliter l'écoulement de la matière sous serre-flan (frottement) et donc de réduire l'énergie consommée durant l'opération de mise en forme.

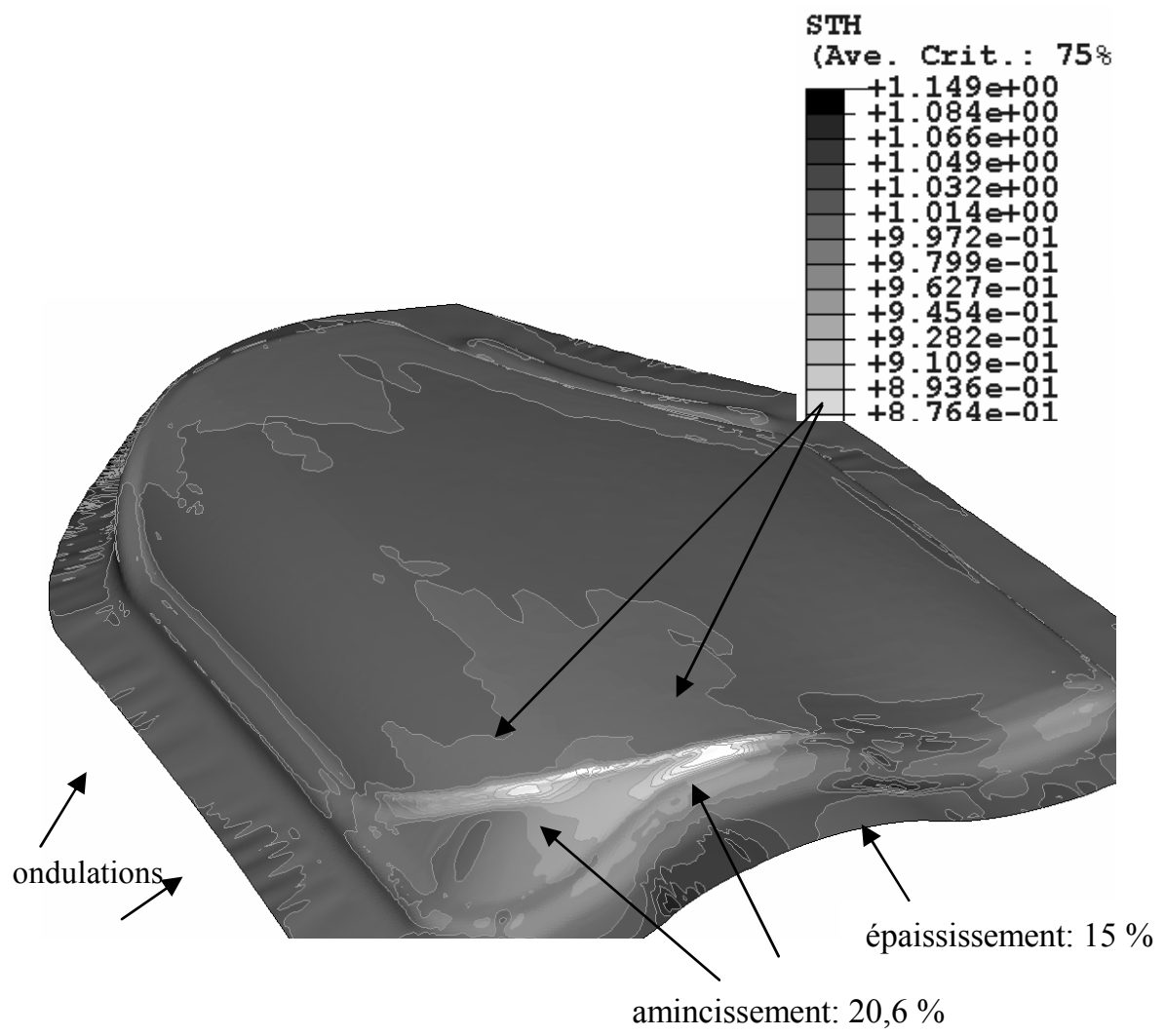

Figure 9. Distribution de l'épaisseur avant optimisation 


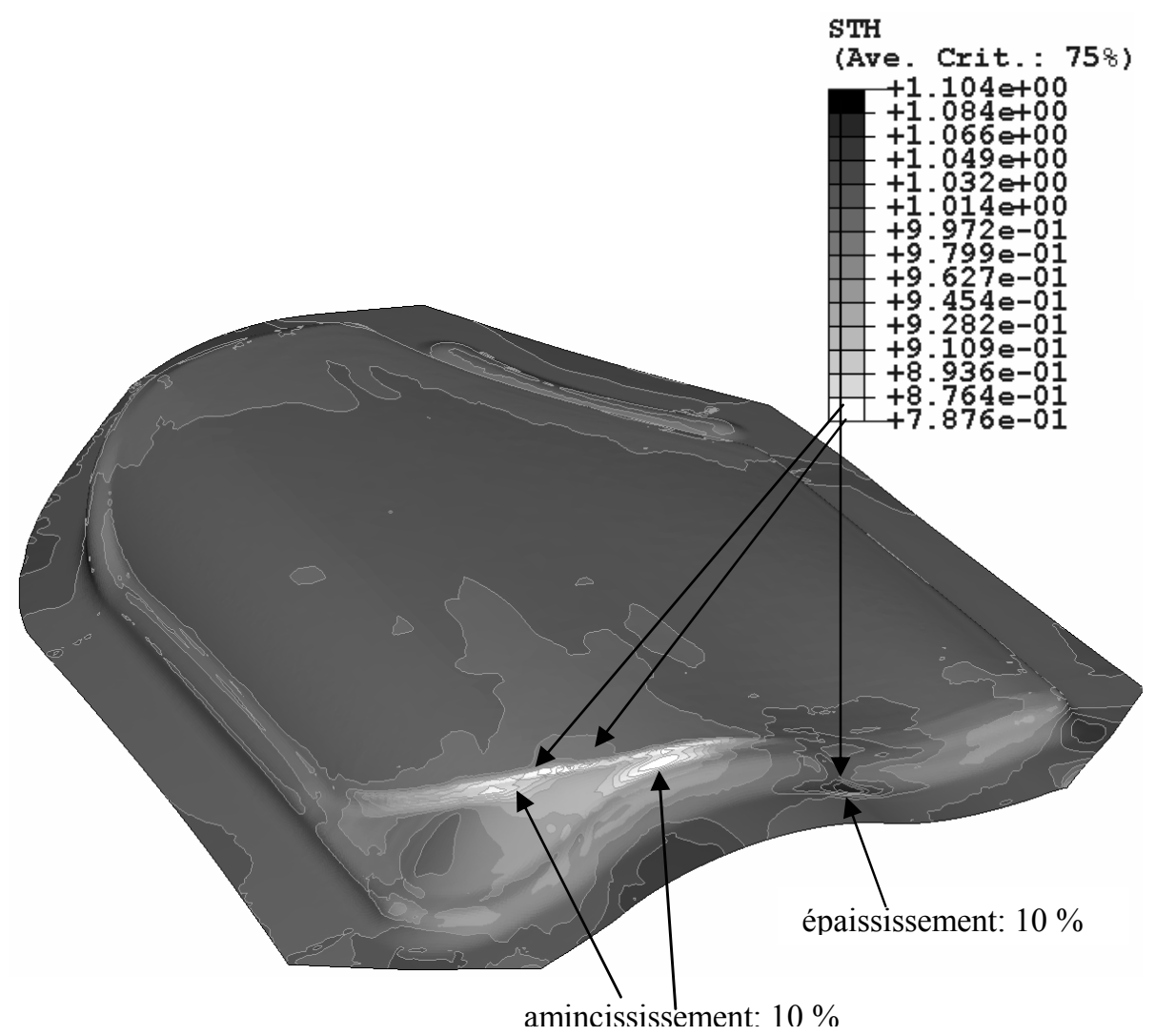

Figure 10. Distribution de l'épaisseur après optimisation

\section{Conclusion}

La simulation numérique et l'optimisation des paramètres des procédés de mise en forme de tôles minces sont deux domaines de recherche et de développement en perpétuelle évolution. Du fait de la croissance rapide des performances des ordinateurs et de la concurrence, les exigences industrielles sont de plus en plus grandes en particulier au niveau de la qualité des pièces embouties et de la rentabilité du procédé d'emboutissage.

Au cours de cette étude, différents aspects d'optimisation et de prédiction des défauts en emboutissage ont été abordés. L'objectif de nos travaux de recherches est de contrôler l'écoulement de la matière sous le serre-flan, par la maîtrise et l'optimisation du profil des efforts sur les serre-flans, afin d'obtenir une pièce « sans défauts » et de réduire le coût de l'opération de mise en forme. En fonction des défauts qui doivent être évités dans la pièce finale, un problème d'optimisation a été 
formulé avec des fonctions limitations exprimant explicitement les critères de qualité choisis (rupture et plissement).

Pour mener à bien la résolution du problème d'optimisation, nous avons utilisé des critères de qualité fiables et une stratégie d'optimisation qui doit tenir compte des spécificités de l'outil de simulation employé, de la non-linéarité du problème d'optimisation et aussi du temps de calcul élevé.

Lors de l'étude menée sur l'application d'emboutissage, il a été indispensable de définir trois fonctions limitations afin d'éviter la rupture et le plissement, sous les serre-flans et dans la partie utile de la pièce. Une répartition optimale et satisfaisante des efforts sur les serre-flans a été obtenue dans un temps de calcul acceptable. Une réelle amélioration de la qualité de la pièce finale a été atteinte.

Malgré l'énorme progrès et les performances des ordinateurs, les méthodes simplifiées (l'approche inverse par exemple (Batoz et al., 2004)) peuvent encore être nécessaires et utiles, dans le cas d'une pièce industrielle assez complexe, au moins dans une première phase d'exploration de l'espace de recherche et d'identification des zones susceptibles d'accueillir l'optimum global. Cette phase facilitera considérablement la localisation de l'optimum avec précision en poursuivant l'étude avec une approche incrémentale. Les travaux associant l'approche inverse (Naceur et al., 2004, Batoz et al., 2004) et l'optimisation doivent ainsi être poursuivis.

Ce travail peut également être poursuivi par l'optimisation de la géométrie d'un serre-flan à rigidité variable et l'optimisation des efforts serre-flans afin de réduire le retour élastique.

Les procédures d'optimisation développées dans cette thèse sont couplées au logiciel généraliste $A B A Q U S$ et il serait très intéressant d'utiliser un autre code incrémental dédié au métier de l'emboutissage (LS-DYNA, PAMSTAMP, AUTOFORM, STAMPACK, RADIOSS) qui permettrait probablement de gagner beaucoup de temps CPU.

\section{Bibliographie}

Barlet O., Contribution à l'optimisation de forme de pièces embouties pour l'industrie de l'automobile, Thèse de doctorat, Université de Technologie de Compiègne, France, 1998.

Batoz J.L., Naceur H., Guo Y.Q., "Formability Predictions in stamping and process parameter optimization based on the inverse Approach Code Fast Stamp", NUMISHEET 2005, Int. Conf. On Numerical Simulation of 3-D Sheet Metal Forming Processes, Detroit, Michigan, USA., August 15-19, 2005.

Ben Ayed L., Delamézière A., Batoz J.L., Knopf-Lenoir C., « Optimisation des efforts serreflan pour l'emboutissage d'une boîte carrée, Contrôle de la striction et du plissement sous serre-flan », Revue Européenne de Mécanique Numérique, 2006, vol. 15/1-2-3, p. 53-65. 
Boudeau N., Gélin J.C., "Necking in sheet metal forming. Influence of macroscopic and microscopic properties of materials", Int. J. Mech. Sci., vol. 42, 2000, n 11, p. 2209-2232.

Boudeau N., Approches méthodologiques, numériques et expérimentales en mise en forme des structures minces, poudre et polymères chargés, Mémoire d'Habilitation à Diriger des Recherches, Université de Franche-Comté, France, 2003.

Brunet M., Mguil S., Morestin F., "Analytical and experimental studies of necking in sheet metal forming processes”, J. Mat. Process. Technol., vol. 80-81, 1998, p. 40-46.

Chu E., Xu Y., “An elastoplastic analysis of flange wrinkling in deep drawing process", Int. J. Mech. Sci., vol. 43, 2001, p. 1421-1440.

Col A., «Emboutissage des tôles: Importance des modes de déformation », Techniques de l’ingénieur, BM 7 510, 2002.

Considère A., "Mémoire sur l'emploi de fer et de l'acier dans les constructions », Annales des Ponts et Chaussées, 9, 574, 1885.

Cordebois J.P., Critères d'instabilité plastique et endommagement ductile en grande déformation (application à l'emboutissage), Thèse d'Etat, Université Paris-VI, France, 1983.

Delamézière A., Contribution à l'optimisation des paramètres du procédé d'emboutissage des tôles minces par l'approche inverse, Thèse de doctorat, Université de Technologie de Compiègne, France, 2002.

Dudzinski D., Molinari A., «Modélisation des instabilités plastiques en emboutissage», Physique et Mécanique de la Mise en Forme des Métaux Ecole d'été d'Oléron, Presses du CNRS, 1990, p. 444-460

Felder E., « Tribologie de l'emboutissage », Techniques de l'Ingénieur, B7535, 1994.

Gantar G., Kuzman K., "Optimization of sheet metal forming processes by the use of numerical simulations”, J. Mat. Process. Technol., vol. 130-131, 2002, p. 54-59.

Gantar G., Kuzman K., "Sensitivity and stability evaluation of deep drawing process", J. Mat. Process. Technol., vol. 125-126, 2002, p. 302-308.

Gantar G., Kuzman K., "Optimization of stamping processes aiming at maximal process stability”, J. Mat. Process. Technol., vol. 167, 2005, p. 237-243.

Ghouati O., Gélin J.C., Lenoir H., "Process optimization in deep drawing”, Revue européenne des éléments finis, vol. 9, $\mathrm{n}^{\circ}$ 1-2-3, 2000, p. 129-149.

Goupy J., Plans d'expérience pour surface de réponse, Dunod, Paris, 1999.

Graf A., Hosford W.F., "Calculations of Forming Limit Diagrams”, Met. Trans., vol. 21A, 1990, p. 87-94.

Häussermann M., "Multipoint-Cushion-Technology Advances and Die Design", New Developments in Sheet Metal Forming, Klaus Siegert (Editor), Fellbach, Germany, 2324 May 2000, p. 341-366.

HKS, Abaqus User's Manuals, version 6.4, 2005. 
Hora P., Tong L., Reissner J., "A prediction method for ductile sheet metal failure in FESimulation”, NUMISHEET'96, $3^{\text {rd }}$. Int. Conf. On Numerical Simulation of 3-D Sheet Metal Forming Processes, Numisheet'96, Dearborn, Michigan, USA, September 29October 3, 1996, p. 252-256.

Hsu C.W., Ulsoy A.G., Demeri M.Y., "Development of process control in sheet metal forming”, J. Mat. Process. Tech., vol. 127, 2002, p. 361-368.

Jansson T., Andersson A., Nilsson L., "Optimization of draw-in for an automotive sheet metal part: An evaluation using surrogate models and response surfaces", J. Mat. Process. Tech., vol. 159, 2005, p. 426-434.

Katayama T., Nakamachi E., Nakamura Y., Ohata T., Morishita Y., Murase H., "Development of process design system for press forming-multi-objective optimization of intermediate die shape in transfer forming", J. Mat. Process. Technol., vol. 155-156, 2004, p. 1564-1570.

Keltz G., Contribution à l'optimisation des procédés d'emboutissage de tôles en construction automobile, Thèse de Doctorat, Université de Franche Comté, 2004.

Kleinermann J.P., Ponthot J.P., "Parameter identification and shape/process optimization in metal forming simulation”, J. Mat. Process. Technol., vol. 139, 2003, p. 521-526.

Kleinermann J.P., Ponthot J.P., Stainier L., "Optimisation of sheet metal forming processes using inverse problems methodology", VII International Conference on Computational Plasticity COMPLAS 2003, Barcelona, Espagne 7-10 Avril 2003, 20 pages sur CD-ROM.

Labergère C., Contributions à la modélisation, à l'optimisation et au contrôle des procédés d'hydroformage de tubes et flans, Thèse de doctorat, Université de Franche-Comté, 2003.

Ladevèze P., Sur la théorie de la plasticité en grande déformation, Rapport interne, $n^{\circ} 9$, LMT/ENSET/Paris-VI, France, 1981.

Lejeune A., Modélisation et simulation de striction et de plissement en emboutissage des tôles minces et hydroformage de tubes minces, Thèse de Doctorat, Université de FrancheComté, France, 2002.

Marciniak Z., Kuczynski K., "Limit strains in the processes of stretch-forming sheet metal", Int. J. Mech. Sci., vol. 9, 1976, p. 609-620.

Molinari A., "Instabilité thermoviscoplastique en cisaillement simple", J. Mécanique Théorique et Appliquée, vol. 4, n 5, 1985, p. 659-684.

Mouric L., Prévision de la striction des tôles élastoplastiques anisotropes embouties, Thèse de Doctorat, Ecole Normale Supérieure de Cachan, France 2002.

Naceur H., Optimisation de forme de structures minces en présence de non linéarités géométriques et matérielles, Thèse de doctorat, Université de Technologie de Compiègne, France, 1998.

Naceur H., Guo Y.Q., Batoz J.L., Knopf-Lenoir C., "Optimization of drawbead restraining forces and drawbead design in sheet metal forming process", Int. J. of Mech. Sc., vol. 43, 2001, p. 2407-2434. 
Naceur H., Breitkopf P., Knopf-Lenoir C., Villon P., « Méthode de surface de réponse pour l'optimisation de forme des surface additionnelles de pièces embouties ", Sixième colloque national en calcul des structures, Giens (Var), France, 20-23 Mai 2003, Tome III, p 215-222,

Naceur H., Delaméziere A., Batoz J.L., Guo Y.Q., Knopf-Lenoir C., "Some improvements on the optimum process design in deep drawing using the inverse approach", $J$. of Mat. Process. Tech., vol. 146, 2004, p. 250-262.

Ohata T., Nakamura Y., Katayama T., Nakamachi E., Nakano K., "Development of optimum process design system by numerical simulation”, J. Mater. Process. Technol., vol. 60, 1996, p. 543-548.

Ohata T., Nakamura Y., Katayama T., Nakamachi E., Omori N., "Improvement of optimum process design system by numerical simulation", J. Mater. Process. Technol., vol. 80-81, 1998, p. 635-641.

Ohata T., Nakamura Y., Katayama T., Nakamachi E., "Development of optimum process design system for sheet fabrication using response surface method", J. Mat. Process. Tech., vol. 143-144, 2003, p. 667-672.

Ponthot J.P., Kleinermann J.P., "A cascade optimization methodology unified for automatic parameter identification and shape/process optimization in metal forming simulation", Computer Methods in Applied Mechanics and Engineering, vol. 195, 2006, John ARGYRIS Memorial Issue, p. 5472-5508.

Siegert K., "Research and Development in the Field of Sheet Metal Forming Technology of the Institute for Metal forming Technology (IFU) of the University of Stuttggart", New Developments in Sheet Metal Forming, Klaus Siegert (Editor), Fellbach, Germany, 2324 May, 2000, p. 277-308.

Swift H. W., Plastic Instability Under Plane Stress, J. Mech. Phys. Solids, V 1, 1952, p. 1-18.

Villon P., Contribution à l'optimisation, Thèse d'état, Université de Technologie de Compiègne, France, 1991.

Xiaoxiang S., Jun C., Yinghong P., Xueyu R., "A new approach of die shape optimization for sheet metal forming processes”, J. Mat. Process. Technol., vol. 152, 2004, p. 35-42. 
